\title{
Multichannel Detection for Wideband Underwater Acoustic CDMA Communications
}

\author{
Milica Stojanovic, Member, IEEE, and Lee Freitag, Member, IEEE
}

\begin{abstract}
Direct-sequence (DS) code-division multiple access (CDMA) is considered for future wideband mobile underwater acoustic networks, where a typical configuration may include several autonomous underwater vehicles (AUVs) operating within a few kilometers of a central receiver. Two receivers that utilize multichannel (array) processing of asynchronous multiuser signals are proposed: the symbol decision feedback (SDF) receiver and the chip hypothesis feedback (CHF) receiver. Both receivers use a chip-resolution adaptive front end consisting of a many-to-few combiner and a bank of fractionally-spaced feedforward equalizers. In the SDF receiver, feedback equalization is implemented at symbol resolution, and receiver filters, including a decision-directed phase-locked loop, are adapted at the symbol rate. This limits its applicability to the channels whose time variation is slow compared to the symbol rate. In a wideband acoustic system, which transmits at maximal chip rate, the symbol rate is down-scaled by the spreading factor, and an inverse effect may occur by which increasing the spreading factor results in performance degradation. To eliminate this effect, feedback equalization, which is necessary for the majority of acoustic channels, is performed in the CHF receiver at chip resolution and receiver parameters are adjusted at the chip rate. At the price of increased computational complexity (there are as many adaptive filters as there are symbol values), this receiver provides improved performance for systems where time variation cannot be neglected with respect to the symbol rate [e.g., low probability of detection (LPD) acoustic systems]. Performance of the two receivers was demonstrated in a four-user scenario, using experimental data obtained over a 2-km shallow-water channel. At the chip rate of $\mathbf{1 9 . 2}$ kilochips per second $(\mathrm{kc} / \mathrm{s})$ with quaternary phase-shift keying (QPSK) modulation, excellent results were achieved at an aggregate data rate of up to $10 \mathrm{~kb} / \mathrm{s}$.
\end{abstract}

Index Terms-Adaptive algorithms, decision-feedback equalization, direct-sequence (DS) spread-spectrum, multichannel combining, space-time processing, underwater acoustic communications, wideband code-division multiple access (CDMA).

\section{INTRODUCTION}

D IRECT-sequence (DS) code-division multiple access (CDMA) is considered as a candidate technique for the future mobile underwater wireless communication networks. Its advantages include efficient use of bandwidth, relaxed synchronization requirements as compared to time-division multiple access (TDMA), inherent multipath diversity, and

Manuscript received December 3, 2004; revised July 23, 2005; accepted November 8, 2005. This work was supported by the Office of Naval Research under the Program "Demonstration of Undersea Autonomous Operation Capabilities and Related Technology Development." Associate Editor: J. Preisig.

M. Stojanovic is with the Massachusetts Institute of Technology, Cambridge, MA 02139 USA (e-mail: millitsa@mit.edu).

L. Freitag is with the Woods Hole Oceanographic Institution, Woods Hole, MA 02543 USA.

Color versions of one or more of the figures in this paper are available online at http://ieeexplore.ieee.org.

Digital Object Identifier 10.1109/JOE.2006.880389 a soft capacity limit [1]. Autonomous underwater vehicles (AUVs), crawlers and gliders, as well as fixed instruments equipped with acoustic modems will be the users of these networks. In a centralized configuration, a fixed or movable base station, mounted on the bottom of the ocean or on a surface buoy, employs an array of hydrophones. A mobile can also have an array of receive elements, possibly a smaller one. At this time, it is practical to conceive of a network consisting of fewer than ten users within a radius of several kilometers. This paper deals with the design of communication methods suitable for multichannel (array) detection of multiuser signals in such a network.

The major obstacles encountered on an underwater channel are extended multipath propagation and rapid time variation, which cause severe frequency-selective fading in a wideband acoustic system. Due to the limited bandwidth, which can be on the order of $10 \mathrm{kHz}$ around a center frequency of several tens of kilohertz for a system operating over few kilometers, a system is designed for transmission at a maximal chip rate, while the achievable bit rate is determined by the spreading factor. The spreading factor is selected to support the desired number of users and provide the necessary quality of performance. At the same time, it is desired that as high a bit rate as possible be achieved. To support several users, say fewer than ten, without excessive bandwidth expansion which would result in the reduction of the information throughput, the spreading factor on the order of ten should be chosen. A higher spreading factor is justified in systems that seek to transmit at low power thus providing low probability of detection (LPD) in addition to supporting multiple users.

Because of the severe frequency-selective distortion caused by multipath propagation, it is beneficial, if not imperative, to use a decision-feedback equalizer over underwater acoustic channels of interest. In particular, multichannel decision-feedback equalization is considered to be the most effective method for detection of nonspread, bandwidth efficient signals in underwater acoustic channels [2]-[4].

The multipath interference, seen as intersymbol interference (ISI) in a nonspread system, appears as interchip interference in a DS spread-spectrum system. If the multipath spread on a shallow-water channel is on the order of $10 \mathrm{~ms}$ (see [2] and references therein for characteristics of acoustic communication channels), it will cause multipath interference that can span a hundred chip intervals. Thus, not only is the interchip interference severe, but the ISI cannot be neglected. In contrast to this situation, ISI in most of the operating DS CDMA radio systems can be neglected [5]. However, as wideband radio systems come into use, ISI emerges as a problem in these systems as well [6]-[9]. A comprehensive overview of both linear and nonlinear 
equalization methods for wideband CDMA radio systems can be found in [7].

In a DS CDMA scenario, the receiver design focuses on integrating an equalization method for suppression of multipath interference with despreading (decorrelation) for suppression of multiple-access interference. A decision-feedback equalizer proposed in [6] for wideband radio channels is based on feedforward filtering performed at chip-resolution, followed by despreading and symbol-resolution decision-feedback equalization. This principle is extended in [7] to multiple-antenna multiuser detection. In the receiver proposed in [8], symbol decisions obtained from a chip-resolution linear equalizer after despreading are used to regenerate the chip sequence. Assuming that it is sufficiently reliable, this sequence is used to perform chip-resolution decision-feedback equalization of the delayed received signal. The equalizer is designed according to the minimum mean square error (mmse) criterion, assuming that the channel is known. An adaptive version of this receiver, which also includes a space-time code, uses a pilot signal to train the recursive least-squares (RLS) equalizer [9]. In all of these receivers, adaptation of the equalizers is performed at the symbol rate, which limits their applicability to those channels that do not change rapidly at the symbol level. Another design, proposed in [10] for rapidly varying underwater acoustic channels, is based on chip-resolution feedforward and feedback equalization, followed by despreading. Adaptation of the equalizer in this receiver is performed at the chip rate, which is made possible by feeding back hypothesized chip values. At the price of increased computational complexity, this receiver gains the ability to perform chip-resolution feedback filtering and fast, chip-rate adaptation. Its performance so far has only been tested in simulation.

In this paper, two types of decision-feedback receivers are proposed which perform decision-feedback equalization and despreading in a multichannel (array) configuration. The first receiver is based on the principle of symbol decision feedback (SDF), while the second receiver is based on the principle of chip hypothesis feedback (CHF). A multichannel combiner [3] and a phase synchronization method are integrated into the receiver structures to enable their operation in realistic mobile underwater acoustic systems.

Performance of the algorithms proposed is demonstrated using experimental data. A four-user CDMA system is considered, operating at 19.2 kilochips per second $(\mathrm{kc} / \mathrm{s})$ at a center frequency of $33 \mathrm{kHz}$. Signals were transmitted between a source and an array of receivers separated by $2.3 \mathrm{~km}$ in water with a depth of $100 \mathrm{~m}$. The array was a 12-element vertical hydrophone array with $0.15-\mathrm{m}$ spacing between the elements. At a spreading factor of 15, 63, and 255, using quaternary phase-shift keying (QPSK) modulation, the aggregate bit rate was $10,2.4$, and $0.6 \mathrm{~kb} / \mathrm{s}$, respectively. Excellent results were achieved owing to the use of multichannel processing. These results demonstrate the possibility to support multiple users in a highly distorted underwater channel, even at minimal processing complexity and low bandwidth expansion.

The SDF and the CHF receivers discussed in this paper are single-user detectors, but draw the necessary processing gain from multichannel combining of the signals observed across the receiver array. Multiuser detection offers performance improve- ment over single-user detection; however, algorithm design is limited by computational complexity. For practical receiver implementation with currently available processing power, multichannel single-user detection offers a viable solution.

The paper is organized as follows. In Section II, receiver algorithms are presented. Experimental results are discussed in Section III. Section IV summarizes the conclusions.

\section{RECEIVER AlgORIthM}

\section{A. System Model}

The complex baseband transmitted signal of the $i$ th user is given by

$$
u_{i}(t)=\sum_{n} d_{i}(n) g_{i}(t-n T), \quad i=1, \ldots I
$$

where $d_{i}(n)$ represents the data sequence, $T$ is the symbol period, and $g_{i}(t)$ is the spreading waveform. The data symbols can take values from an arbitrary quadratic-amplitude modulation (QAM) or phase-shift keying (PSK) alphabet. The spreading waveform is given by

$$
g_{i}(t)=\sum_{l=0}^{L-1} p_{i}(l) g\left(t-l T_{c}\right)
$$

where $p_{i}(l), l=0, \ldots L-1$, is the chip sequence of the $i$ th spreading code, $T_{c}$ is the chip interval, and $g(t)$ is the transmitter shaping pulse. The code period is equal to the symbol duration $L T_{c}=T$. The chip sequence can be complex or real-valued $( \pm 1)$. The transmitted signal can alternatively be expressed as

$$
u_{i}(t)=\sum_{k} q_{i}(k) g\left(t-k T_{c}\right)
$$

where $q_{i}(k)$ is the modulated chip sequence, and

$$
q_{i}(n L+l)=d_{i}(n) p_{i}(l), \quad l=0, \ldots L-1 \quad \forall n .
$$

The signals of different users travel through different, multipath-distorted channels, and arrive at a common receiver. In Sections II-B and II-C, we describe two receivers, one based on SDF, and another based on CHF.

\section{B. SDF Receiver}

The block diagram of the SDF receiver is shown in Fig. 1. The same receiver structure is used for each desired user, whose index $i$ we drop for simplicity of notation, i.e., $d(n)=d_{i}(n)$ and $p(l)=p_{i}(l)$ is assumed in what follows. The received complex baseband signal observed at the $k$ th array element is denoted by $v_{k}(t), k=1, \ldots K$. The signals are sampled at the Nyquist or higher rate, resulting in $N_{s}$ samples per chip interval $T_{c}$. The $K$ received signals are first spatially combined to form $P \leq K$ inputs for the feedforward filters. In doing so, no temporal processing is involved, as this will be accomplished by the multichannel equalizer that follows. The goal of spatial combining is to form a reduced number of channels $P$ for subsequent processing, thus keeping the complexity manageable when there is a large number of input channels $K$. The weights of the spatial combiner are optimized jointly with those of the multichannel equalizer, thus ensuring broadband processing necessary for the 


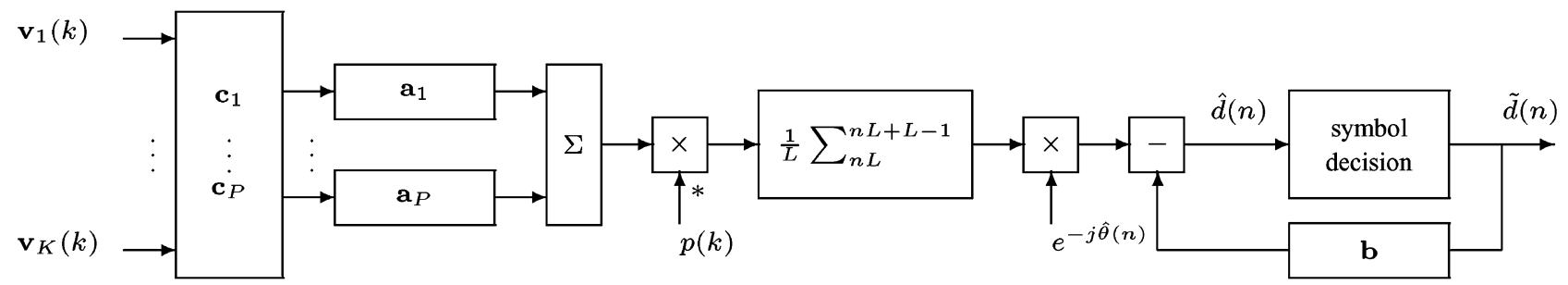

Fig. 1. DS receiver based on multichannel (many-to-few) combining and symbol decision feedback equalization. Symbol decisions are also used to update the combiner, the equalizer filters, and the phase estimate. The feedforward filters have fractionally chip-spaced taps. The feedback filter has symbol-spaced taps. Receiver parameters are updated at the symbol rate. Adaptive algorithm is driven by the symbol error $\tilde{d}(n)-\hat{d}(n)$.

wideband signals at hand, as well as a good tradeoff between performance and complexity [3].

The combiner coefficients are arranged into $K$-element column vectors $\mathbf{c}_{p}, p=1, \ldots P$. The feedforward filters are fractionally spaced at $T_{c} / N_{s}$, and each has $N$ coefficients arranged in a column vector $\mathbf{a}_{p}, p=1, \ldots P$. The temporal samples of the $k$ th receive element are arranged into an $N$-element vector

$$
\begin{aligned}
\mathbf{v}_{k}(n, l)=\mathbf{v}_{k}(n L+l) & =\left[\ldots v_{k}\left(n T+l T_{c}+T_{s}\right),\right. \\
& \left.v_{k}\left(n T+l T_{c}\right), v_{k}\left(n T+l T_{c}-T_{s}\right) \ldots\right]^{T}
\end{aligned}
$$

where the time index $(n, l)$ refers to the $l$ th chip of the $n$th data symbols. It is assumed that coarse time-synchronization is performed such that the vectors $\mathbf{v}_{k}(n, l), l=0, \ldots L-1$ contain significant contribution of the $n$th transmitted data symbol $d(n)$. The signal vectors of all receive elements form the signal matrix

$$
\mathbf{V}(n, l)=\mathbf{V}(n L+l)=\left[\begin{array}{c}
\mathbf{v}_{1}^{T}(n, l) \\
\vdots \\
\mathbf{v}_{K}^{T}(n, l)
\end{array}\right], \quad l=0, \ldots L-1 .
$$

The outputs of the feedforward equalizers are summed, and this signal is despread using the desired user's code and synchronized using a phase estimate $\hat{\theta}(n)$. In many practical cases, it is sufficient to use a single-carrier phase estimate, and we do so for the sake of computational efficiency. Extension to multiple carrier estimates (one per input channel, or one per equalizer input) is straightforward. The feedback filter has $M$ coefficients arranged into a vector $\mathbf{b}$. During the detection of the $n$th data symbol, the feedback filter operates on previous symbols, $\tilde{\mathbf{d}}(n-1)=[\tilde{d}(n-1) \ldots \tilde{d}(n-M)]^{T}$, which represent the known data symbols during training, and decisions thereafter. The estimate of the $n$th data symbol is obtained as

$$
\hat{d}(n)=\frac{1}{L} \sum_{l=0}^{L-1} p^{*}(l) \sum_{p=1}^{P} \mathbf{c}_{p}^{\prime} \mathbf{V}(n, l) \mathbf{a}_{p}^{*} e^{-j \hat{\theta}(n)}-\mathbf{b}^{\prime} \tilde{\mathbf{d}}(n-1)
$$

where prime denotes conjugate transpose. This expression defines the dependence of the data estimate on all the receiver parameters - the combiner weights, the feedforward filters, the feedback filter, and the phase estimate. These parameters are jointly adjusted to minimize the mean square error (mse) in data detection. The adaptive algorithm is driven by a single error $e(n)=\tilde{d}(n)-\hat{d}(n)$.
To arrive at an adaptive algorithm for the equalizer, (6) is rewritten as

$$
\hat{d}(n)=\left[\begin{array}{ll}
\mathbf{a}^{\prime} & \mathbf{b}^{\prime}
\end{array}\right]\left[\begin{array}{c}
\mathbf{x}_{a}(n) e^{-j \hat{\theta}(n)} \\
-\tilde{\mathbf{d}}(n-1)
\end{array}\right]
$$

where $\mathbf{a}^{\prime}=\left[\mathbf{a}_{1}^{\prime} \ldots \mathbf{a}_{P}^{\prime}\right]$ is the vector of all feedforward equalizer coefficients, and

$$
\mathbf{x}_{a}(n)=\frac{1}{L} \sum_{l=0}^{L-1} p^{*}(l)\left[\begin{array}{c}
\mathbf{V}^{T}(n, l) \mathbf{c}_{1}^{*} \\
\vdots \\
\mathbf{V}^{T}(n, l) \mathbf{c}_{K}^{*}
\end{array}\right] .
$$

Once the data estimate is expressed as an inner product (7) between a filter vector and its equivalent input signal, adaptation of the equalizer is specified by

$$
\left[\begin{array}{l}
\mathbf{a}(n+1) \\
\mathbf{b}(n+1)
\end{array}\right]=\left[\begin{array}{l}
\mathbf{a}(n) \\
\mathbf{b}(n)
\end{array}\right]+A_{e}\left(\left[\begin{array}{c}
\mathbf{x}_{a}(n) e^{-j \hat{\theta}(n)} \\
-\tilde{\mathbf{d}}(n-1)
\end{array}\right], e(n)\right)
$$

where $A_{e}$ is the chosen adaptive algorithm whose first argument denotes the input signal vector and the second argument denotes the error. For example, if the least mean square (LMS) algorithm is chosen for computational simplicity, we have that

$$
\left[\begin{array}{l}
\mathbf{a}(n+1) \\
\mathbf{b}(n+1)
\end{array}\right]=\left[\begin{array}{c}
\mathbf{a}(n) \\
\mathbf{b}(n)
\end{array}\right]+\mu_{e}\left[\begin{array}{c}
\mathbf{x}_{a}(n) e^{-j \hat{\theta}(n)} \\
-\tilde{\mathbf{d}}(n-1)
\end{array}\right] e^{*}(n)
$$

where $\mu_{e}$ is the equalizer step size.

To define an adaptive algorithm for the combiner coefficients, the data symbol estimate (6) is expressed as

$$
\hat{d}(n)=\mathbf{c}^{\prime} \mathbf{x}_{c}(n) e^{-j \hat{\theta}(n)}-\mathbf{b}^{\prime} \tilde{\mathbf{d}}(n-1)
$$

where $\mathbf{c}^{\prime}=\left[\mathbf{c}_{1}^{\prime} \ldots \mathbf{c}_{P}^{\prime}\right]$ is the composite vector of all the combiner coefficients, and

$$
\mathbf{x}_{c}(n)=\frac{1}{L} \sum_{l=0}^{L-1} p^{*}(l)\left[\begin{array}{c}
\mathbf{V}(n, l) \mathbf{a}_{1}^{*} \\
\vdots \\
\mathbf{V}(n, l) \mathbf{a}_{P}^{*}
\end{array}\right]
$$

Adaptation of the combiner is now specified by

$$
\mathbf{c}(n+1)=\mathbf{c}(n)+A_{c}\left(\mathbf{x}_{c}(n) e^{-j \hat{\theta}(n)}, e(n)\right) .
$$

The adaptive algorithm $A_{c}$ can be chosen independently of the algorithm $A_{e}$. Updating of the equalizer (9) and of the spatial combiner (13) is performed once per symbol interval. 
To obtain the phase estimate $\hat{\theta}(n)$, a second-order decisiondirected digital phase-locked loop (PLL) is employed

$$
\hat{\theta}(n+1)=\hat{\theta}(n)+K_{1} \psi(n)+K_{2} \sum_{m \leq n} \psi(m)=\hat{\theta}(n)+\operatorname{PLL}(\psi(n))
$$

where $\psi(n)$ is the PLL input signal, and $K_{1}, K_{2}$ are the PLL tracking constants. The PLL input is defined as

$$
\psi(n)=-\frac{1}{2} \frac{\partial\left|e^{2}(n)\right|}{\partial \hat{\theta}}=\operatorname{Im}\left\{x(n) e^{-j \hat{\theta}(n)} e^{*}(n)\right\}
$$

where

$$
x(n)=\mathbf{c}^{\prime} \mathbf{x}_{c}(n)=\mathbf{a}^{\prime} \mathbf{x}_{a}(n) .
$$

The algorithm can be initialized with $\hat{\theta}(0)=0, \mathbf{a}=\mathbf{0}$, $\mathbf{b}=\mathbf{0}$, and $\mathbf{c}_{p}=\mathbf{0}_{p}$, an all-zero vector except for the $p$ th element which is 1 . The total number of coefficients that are adaptively adjusted is $K P$ for the combiner and $P N+M$ for the equalizer. Note that the feedback filter length $M$ is determined by the multipath spread measured in symbols.

An algorithm that strives to minimize the overall mse with respect to a number of parameters by calculating individual mmse solutions for each parameter assuming that the others are held constant belongs to a class of coordinate descent optimization procedures. In general, convergence of these algorithms to a global minimum is not guaranteed; however, they have been successfully used in a variety of practical problems, including multiuser detection in CDMA systems (see, e.g., [11] and references therein).

Adaptation of the SDF receiver parameters is performed at the symbol rate. As symbol decisions become available at the end of the despreading period, they are fed back to the equalizer, and also used to generate the error that drives the adaptive algorithm. The receiver parameters are thus updated every $L$ chip intervals. At a fixed chip rate (normally chosen maximal within the available acoustic bandwidth) this means that by increasing the spreading factor the symbol duration increases, thus allowing for a greater change to occur in the channel between successive updates. Hence, while the interference suppression capability improves with increased spreading factor, the channel tracking capability degrades. In conditions of rapid channel variation, which may arise in mobile acoustic channels, this may lead to a counterproductive effect where by increasing the spreading gain results in an overall degradation of system performance. The Doppler frequency, given by $f_{d}=f_{c} v / c$ for a carrier frequency $f_{c}$ and a vehicle speed $v$, can become quite large as compared to the symbol rate because of the low speed of sound (nominal value is $c=1500 \mathrm{~m} / \mathrm{s}$ ). For example, at $f_{c}=33 \mathrm{kHz}$, and a chip rate of $R_{c}=19.2 \mathrm{kc} / \mathrm{s}$, which are the values used for the experimental system, the normalized Doppler frequency is $f_{d} T=0.001 v L$. The speed of an AUV is on the order of few meters per second, and, hence, the factor $f_{d} T$ can become large even at a moderate spreading factor $L$. The channel variation over one symbol interval then becomes too large to be tracked by an adaptive algorithm, causing the SDF receiver to deteriorate in performance, or eventually to fail. This fact serves as a motivation to investigate a different type of adaptive receiver, in which parameter updates are performed at the chip rate rather than the symbol rate.

\section{CHF Receiver}

Adaptive filtering in the CHF receiver is performed entirely at the chip rate, as if there were no spreading and the modulated chip sequence represented the only data stream. The feedback filter is chip-spaced, and all the receiver parameters are updated at the chip rate. Despreading and symbol decision are performed separately, using the estimated chip sequence. However, to enable chip-rate filtering and adaptation, chip decisions must be available before despreading is complete and the symbol decision is made. Hence, the symbol decision is not available at the time when it is needed to generate the current chip decision. To overcome this problem, a set of hypotheses is generated for each symbol interval. The principle of hypothesis feedback equalization, given in [10] for the single-channel receiver configuration, remains the same for the multichannel configuration, and we only briefly summarize its key points before describing the receiver algorithm.

Each hypothesis corresponds to one possible value of the transmitted data symbol. For QPSK, $H=4$ hypotheses are made at the beginning of each symbol interval, denoted by superscript $h$ for the desired user $i: d_{i}^{h}(n)=e^{j(2 h-1) \pi / 4}, h=$ $1, \ldots 4$. Under each hypothesis $h$, the modulated chip sequence is known for the $L$ chips of the $n$th symbol interval

$$
q_{i}^{h}(n L+l)=d_{i}^{h}(n) p_{i}(l), \quad l=0, \ldots, L-1 .
$$

Using the resulting four sequences, four adaptive algorithms ( $H$ in general) are run in parallel for $L$ iterations. The output of the algorithm corresponding to hypothesis $h$ is the sequence of chip estimates $\hat{q}_{i}^{h}(n L+l), l=0, \ldots, L-1$. These estimates are used by the despreader to form the symbol estimates

$$
\hat{d}_{i}^{h}(n)=\frac{1}{L} \sum_{l=0}^{L-1} \hat{q}_{i}^{h}(n L+l) p_{i}^{*}(l), \quad h=1, \ldots H .
$$

The data estimate of the correct hypothesis should most closely resemble the hypothesized symbol value. Thus, the squared error

$$
E_{i}^{h}(n)=\left|d_{i}^{h}(n)-\hat{d}_{i}^{h}(n)\right|^{2}
$$

can be used to select the best hypothesis as

$$
\tilde{h}=\arg \min _{h=1, \ldots H} E_{i}^{h}(n) .
$$

The corresponding symbol decision is

$$
\tilde{d}_{i}(n)=d_{i}^{\tilde{h}}(n) .
$$

The block diagram of the CHF receiver is shown in Fig. 2. The front end of the receiver is identical to that of the SDF receiver. Dropping the desired user's index $i$, the chip estimate at the output of the front section is expressed as

$$
\hat{q}^{h}(k)=\underbrace{\sum_{p=1}^{P} \mathbf{c}_{p}^{h^{\prime}} \mathbf{V}(k) \mathbf{a}_{p}^{h *} e^{-j \hat{\theta}^{h}(k)}}_{x^{h}(k)}-\mathbf{b}^{h^{\prime}} \tilde{\mathbf{q}}^{h}(k-1) .
$$




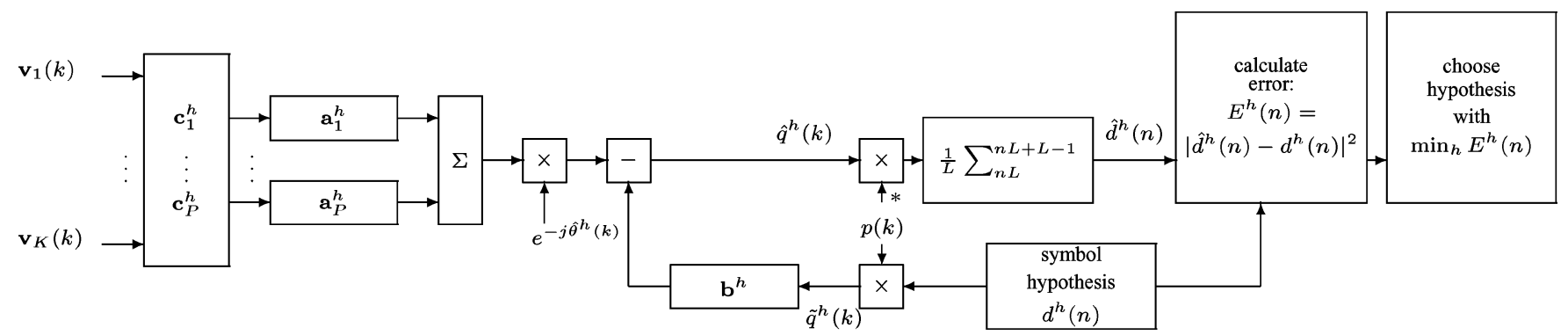

Fig. 2. DS receiver based on multichannel (many-to-few) combining and chip hypothesis feedback equalization. One symbol worth of chip hypotheses are also used to update the combiner, the equalizer filters, and the phase estimate. The feedforward filters have fractionally chip-spaced taps. The feedback filter has chipspaced taps. Receiver parameters are updated at the chip rate. Adaptive algorithm is driven by the chip error $\tilde{q}^{h}(k)-\hat{q}^{h}(k)$.

The combiner, the equalizer, and the phase estimate corresponding to hypothesis $h$ are labeled accordingly. Note that in this receiver the phase estimate is obtained every chip interval, and that the feedback filter operates on the previous chips, $\tilde{\mathbf{q}}^{h}(k-1)=\left[\tilde{q}^{h}(k-1) \ldots \tilde{q}^{h}(k-M)\right]^{T}$. The chips stored at time $k=n L+l$ in the feedback filter contain $l$ hypothesized values as the most recent entries, while the remaining $M-l$ values correspond to the spreading code modulated by the previous symbol decisions $\tilde{d}(n-1), \tilde{d}(n-2)$, etc.

Optimization of the CHF receiver is based on the mmse criterion. Adaptation of all the receiver parameters is now driven by the chip estimation error $e_{q}^{h}(k)=\tilde{q}^{h}(k)-\hat{q}^{h}(k)$. Expression (22) shows the dependence of the chip estimate on all the receiver parameters. It can be arranged in the inner product forms that reflect dependence on the composite equalizer and combiner vectors. These forms define the equivalent inputs for adaptive algorithms.

Application of an adaptive algorithm $A_{e}$ to the equalizer coefficients is defined by

$$
\begin{aligned}
& {\left[\begin{array}{c}
\mathbf{a}_{1}^{h}(k+1) \\
\vdots \\
\mathbf{a}_{P}^{h}(k+1) \\
\cdots-\cdots \\
\mathbf{b}^{h}(k+1)
\end{array}\right]=\left[\begin{array}{c}
\mathbf{a}_{1}^{h}(k) \\
\vdots \\
\mathbf{a}_{P}^{h}(k) \\
\cdots-\cdot \\
\mathbf{b}^{h}(k)
\end{array}\right]} \\
& \left.+A_{e}\left(\left[\begin{array}{c}
\mathbf{V}^{T}(k) \mathbf{c}_{1}^{h *}(k) \\
\vdots \\
\mathbf{V}^{T}(k) \mathbf{c}_{P}^{h *}(k)
\end{array}\right] e^{-j \hat{\theta}^{h}(k)}\right], e_{q}^{h}(k)\right) .
\end{aligned}
$$

Similarly, application of an adaptive algorithm $A_{c}$ to the combiner coefficients is defined by

$$
\begin{aligned}
& {\left[\begin{array}{c}
\mathbf{c}_{1}^{h}(k+1) \\
\vdots \\
\mathbf{c}_{P}^{h}(k+1)
\end{array}\right]=} {\left[\begin{array}{c}
\mathbf{c}_{1}^{h}(k) \\
\vdots \\
\mathbf{c}_{P}^{h}(k)
\end{array}\right] } \\
&+A_{c}\left(\left[\begin{array}{c}
\mathbf{V}(k) \mathbf{a}_{1}^{h *}(k) \\
\vdots \\
\mathbf{V}(k) \mathbf{a}_{P}^{h *}(k)
\end{array}\right] e^{-j \hat{\theta}^{h}(k)}, e_{q}^{h}(k)\right)
\end{aligned}
$$

Updating of the equalizer (23) and of the spatial combiner (24) is performed once per chip interval.
The phase estimator is defined by

$$
\hat{\theta}^{h}(k+1)=\hat{\theta}^{h}(k)+\operatorname{PLL}\left(\operatorname{Im}\left\{x^{h}(k) e^{-j \hat{\theta}^{h}(k)} e_{q}^{h^{*}}(k)\right\}\right)
$$

where $\operatorname{PLL}(\cdot)$ denotes the filtering operation similar to (14). Note that the tracking constants for the chip-rate updating can be chosen lower than those for the symbol-rate updating, as there is less change between successive signal observations.

Algorithm initialization is the same as for the SDF receiver. At the end of each symbol interval, the best hypothesis is selected. The combiner coefficients, the equalizer coefficients (together with the feedback filter content), and the phase estimate corresponding to the winning hypothesis are used as initial values for the new symbol interval. Note that the feedback filter length $M$ should now be chosen in accordance with the multipath spread measured in chips, not symbols.

The implementation complexity of the CHF receiver is at least four times that of the SDF receiver for a quaternary modulation method (twice for binary). This price is paid to recover the performance on a rapidly varying channel. Because tracking is performed at the chip-rate rather than the symbol-rate, the spreading factor should no longer influence the quality of adaptive estimation. In this manner, the inverse effect of spreading factor on the performance quality is eliminated, ensuring that higher spreading factor results in better multiple-access interference suppression.

\section{EXPERIMENTAL RESULTS}

\section{A. Signal Design and the Experiment}

DS CDMA signals were designed for four users. Kasami spreading codes of length 15, 63, and 255 were used (there are 4,8 , and 16 codes of these lengths, respectively). The modulation method was QPSK, and the same binary sequence was used for spreading the in-phase and quadrature components. Transmission was organized in packets of equal duration, each containing a channel probe followed by a $50-\mathrm{ms}$ pause, and the data block. The channel probe was a $100-\mathrm{ms}$ frequency sweep spanning the bandwidth between $21 \mathrm{~Hz}$ and $45 \mathrm{kHz}$. The carrier frequency was $f_{c}=33 \mathrm{kHz}$. The data block begins with an acquisition sequence, followed by the information-bearing signal. Pulse shaping was performed using square-root raised-cosine pulses with roll-off factor 0.25 . The chip rate was $R_{c}=19.2 \mathrm{kc} / \mathrm{s}$. The corresponding bit rates, obtained with the spreading factor of 15,63 , and 255 , are 2500 , 


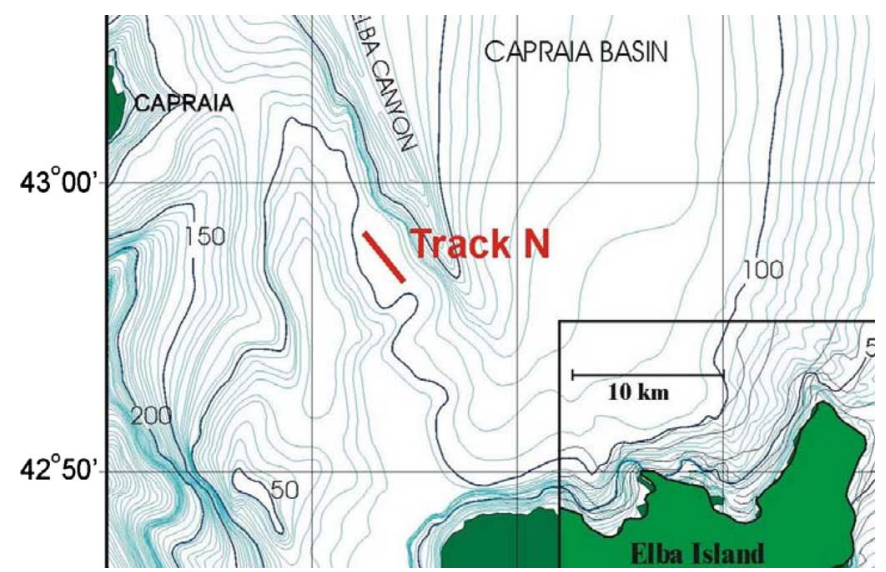

Fig. 3. Experiment location north of Elba. The transmitter and the receiver were deployed from ships positioned along the indicated track near $43^{\circ} \mathrm{N}$.

600 , and $150 \mathrm{~b} / \mathrm{s}$ per user, resulting in an aggregate bit rate of $10,2.4$, and $0.6 \mathrm{~kb} / \mathrm{s}$, respectively.

Passband signals, generated at a sampling rate of $96 \mathrm{kHz}$, were stored for later transmission. Transmission was organized sequentially, according to the following schedule: $L=15$ group, user 1, 2, 3, 4; $L=63$ group, user 1, 2, 3, 4; and $L=$ 255 group, user $1,2,3,4$. The packets within one group were separated by $24 \mathrm{~s}$, and there was a pause of about $1.5 \mathrm{~min}$ between groups.

The experiment was conducted by the Woods Hole Oceanographic Institution, Woods Hole, MA, in the fall of 2003, near the Elba Island in Italy. Location of the experiment is shown in Fig. 3. This experiment was part of a larger experimental effort organized off the NATO Undersea Research Centre, La Spezia, Italy, ship Alliance, which was positioned at $42.92^{\circ} \mathrm{N}, 9.99^{\circ} \mathrm{E}$. The range between the transmitter and receiver was $2.3 \mathrm{~km}$, in water depth of about $100 \mathrm{~m}$. The source was submerged from the transmitting ship to a depth of $20 \mathrm{~m}$, and the signals were received at a 12-element vertical array, submerged from the receiving ship to a depth of $30 \mathrm{~m}$. The array elements were equally spaced at $d=0.15 \mathrm{~m}$. A drift in the transmission range of about $150 \mathrm{~m}$ was observed over the course of a 15-min recording. The recorded signals were stored for later processing.

All the signal processing was performed in baseband, after demodulation using nominal carrier and downsampling to $N_{s}=$ 2 samples per chip, which suffices for the signal bandlimited within $\pm 1 / T_{c}$. The multiuser test signals were constructed by adding a desired number of individual users' signals.

\section{B. Channel Characteristics}

Channel impulse responses, whose magnitudes are shown in Figs. 4-6, were obtained by matched filtering to the frequency sweep probe. Fig. 4 shows the responses obtained during transmission of the $L=15$ group of signals. The first column contains six responses observed at six of the array elements (every other) at the beginning of packet from user 1. The second column represents the responses at the beginning of packet from user 2, and so on. Hence, shown vertically is the spatial variation of the channel response across the array, while the horizontal sequence of responses shows their variation in time. Clearly, both types of variation are present in the channel. The channel exhibits strong multipath and a moderate delay spread of several milliseconds. Nonetheless, this delay spread spans tens of chip intervals in the wideband system considered. In most of the cases, there are two pronounced multipath components, separated by approximately 12.5 chips. These components are followed by distant echoes of decaying energy. The two pronounced multipath arrivals can be associated with the direct path and the surface reflection. Using the geometry of the system to calculate the interarrival time of these two components, one obtains $0.5 \mathrm{~ms}$, a value that roughly coincides with the delay between the two principal components. The angle of arrival of the surface reflection obtained from the system geometry is about $1.5^{\circ}$, which is within the angular spread $\alpha<\sin ^{-1}\left(c T_{c} /(K-1) d\right) \approx 2.7^{\circ}$, required for coherent array combining, i.e., for the signal delay across the array to be less than one chip interval.

Channel responses of Fig. 5, observed during transmission of the $L=63$ group of signals (a minute or so after the $L=15$ group) show a most interesting behavior. Looking at the first column, it is observed that the two principal arrivals vary in strength as the depth changes, such that the early arrival is perceived as strongest at some array elements, while the late arrival appears as strongest at other elements. Looking at a given array element, say element 1 , across time, a similar observation is made. The time variation of the channel causes the early arrival to appear as strongest at some times, yielding to the late arrival at other times. Clearly, the channel is rapidly varying even in the absence of intentional motion. The fact that the strongest arrival is not well defined bears important implications on the receiver performance when initial time synchronization is based on locating the maximum of the probe-matched filter output. Assuming that this would be the case in a working system, all the signals were time-synchronized in accordance with the channel response plots. These signals were then processed by the data detection algorithms. Hence, depending upon its space/time position, a signal may appear to the equalizer as causal or anticausal.

Fig. 6 shows the responses observed during transmission of the $L=255$ group of signals. Similar behavior is observed within this data set. User 1 has a relatively mild channel, user 2 has strong, anticausal multipath, user 3 has strong causal multipath, while user 4 again has strong anticausal multipath. Judging by these snapshots, the coherence time $\Delta t_{c}$ of the channel can be estimated to be on the order of a second. This value implies a normalized Doppler spread $T / \Delta t_{c}$ on the order of $10^{-5} L$, which is well within the tracking capabilities of chip-rate adaptive algorithms considered, but approaches the limit of symbolrate adaptation capability as the spreading factor increases to more than a hundred.

\section{Performance Results}

The SDF and the CHF receiver algorithms were applied to the complex baseband received signals. A multiuser test signal was constructed by adding the signals of all four users. The signals were added at equal power and asynchronously. The desired user is arbitrarily chosen as user 1, while the interfering users' 

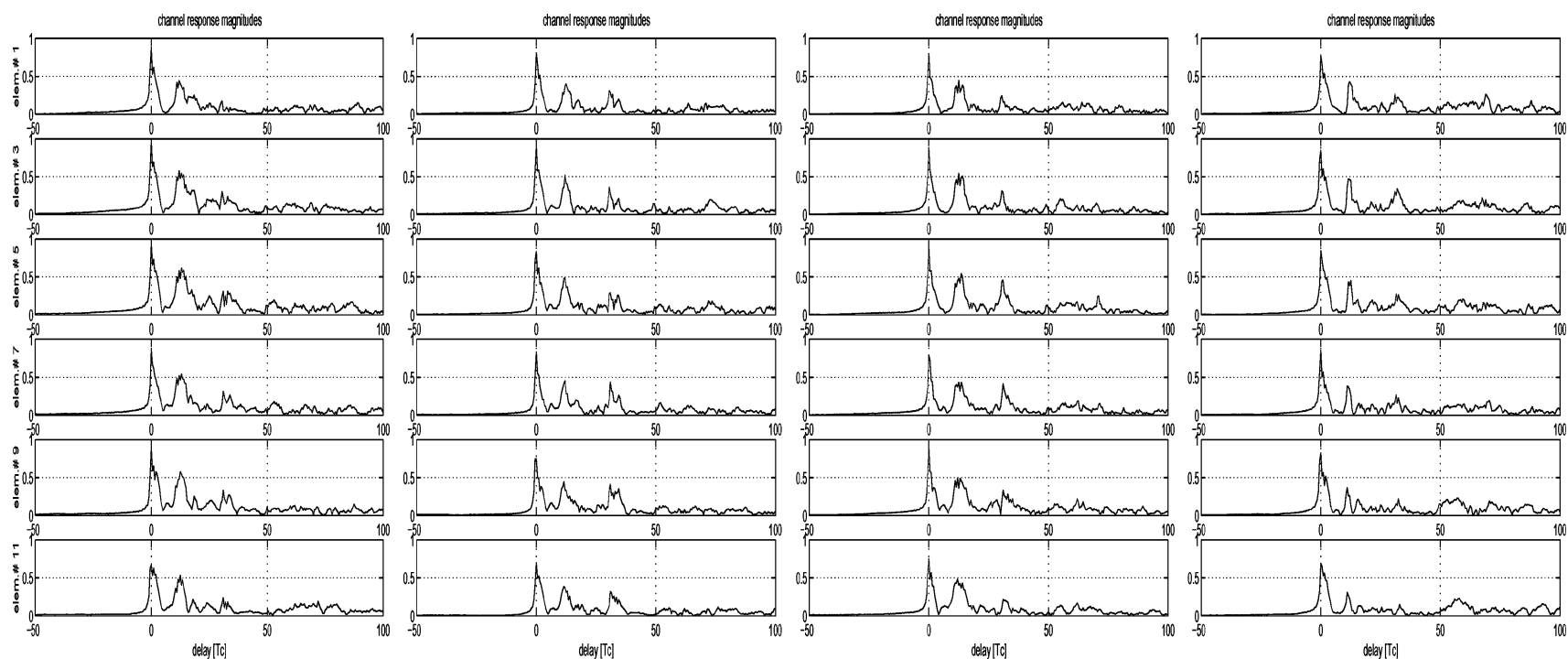

Fig. 4. Channel responses in space and time. Estimates are obtained by matched filtering to the frequency sweep channel probe, which is transmitted before each data packet. Each column shows the magnitudes of the channel responses estimated across the array, for every other of the 12 array elements spaced at $0.15 \mathrm{~m}$. The elements are numbered top to bottom, and each response is shown as a function of delay measured in chip intervals $\left(T_{c}=1 / R_{c} \approx 0.05 \mathrm{~ms}\right)$. The four columns correspond to the four users, whose packets are transmitted in sequence, separated by $24 \mathrm{~s}$. (The signals are later added to form the multiuser test signal.) This figure shows the channel responses recorded during transmission of signals with $L=15$ spreading factor.
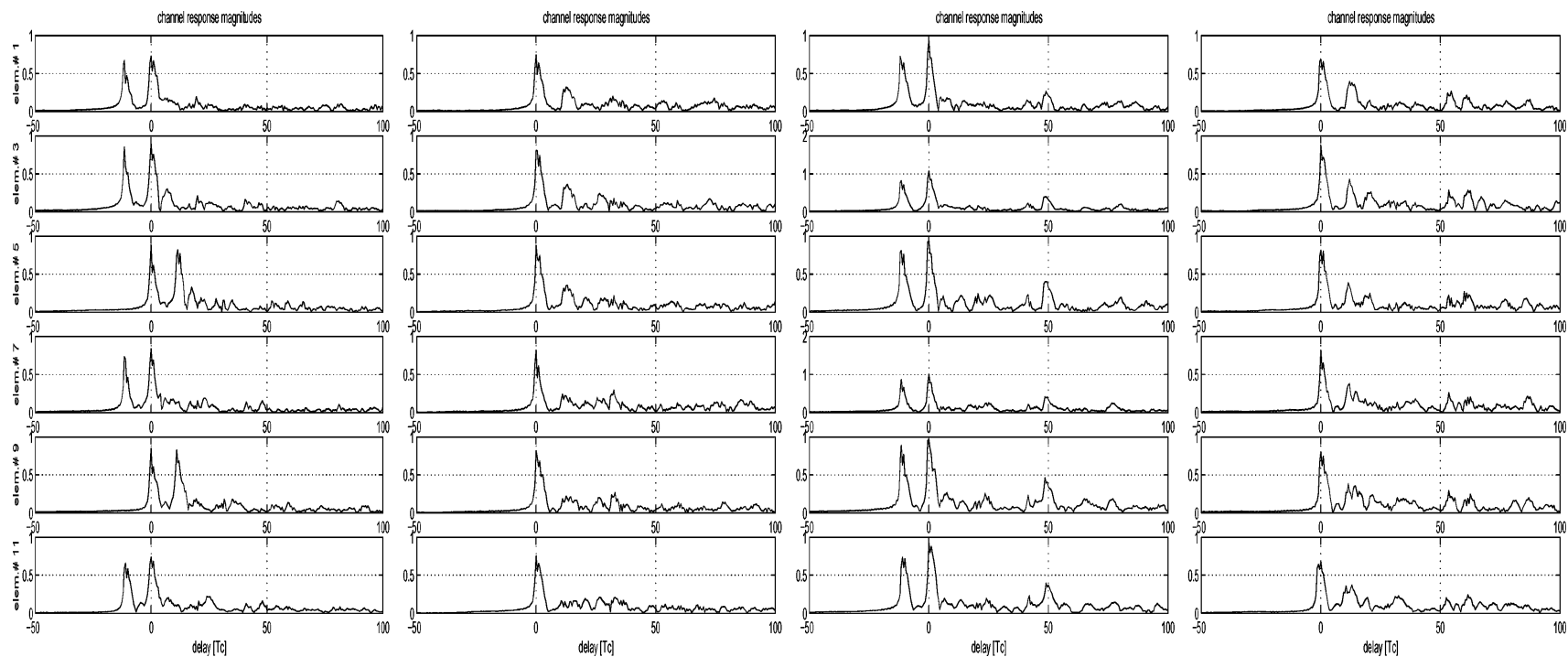

Fig. 5. Channel responses in space and time. Estimates are obtained by matched filtering to the frequency sweep channel probe, which is transmitted before each data packet. Each column shows the magnitudes of the channel responses estimated across the array, for every other of the 12 array elements spaced at $0.15 \mathrm{~m}$. The elements are numbered top to bottom, and each response is shown as a function of delay measured in chip intervals $\left(T_{c}=1 / R_{c} \approx 0.05 \mathrm{~ms}\right)$. The four columns correspond to the four users, whose packets are transmitted in sequence, separated by $24 \mathrm{~s}$. (The signals are later added to form the multiuser test signal.) This figure shows the channel responses recorded during transmission of signals with $L=63$ spreading factor.

signals were arbitrarily delayed by 8,4 , and 2 chip intervals with respect to the desired user's signal.

The decision-feedback equalizer size was determined from the estimated channel responses so as to span the significant delay spread of the channel currently observed. The SDF receiver used an RLS combiner and an LMS equalizer. The CHF receiver used the LMS algorithm for both the combiner and the equalizer. This choice was made to offset the complexity of hypothesis feedback detection, where four adaptations are carried out in parallel. The SDF receiver benefits greatly from the RLS combiner which provides fast convergence. The LMS step sizes and the RLS forgetting factors were determined experimentally and in accordance with the number of receiver coefficients. The
PLL proportional tracking constant $K_{1}$ was set to 0.0005 for the chip-rate filtering, and scaled by the spreading factor $L$ for symbol-rate processing. The PLL integral tracking constant was set to $K_{2}=K_{1} / 10$. Various number of array elements $K$ and equalizer branches $P$ were tested. Here, we report on a set of results that utilized all 12 elements of the array. Three cases are studied later, corresponding to the spreading factor of 15,63 , and 255. The data packet in each case contains the same number of chips, corresponding to 4000,1000 , and 250 symbols, respectively.

Figs. 7 and 8 show performance results obtained with the spreading factor $L=15$. Shown in each figure are the squared symbol error and the phase estimate over the packet duration, 

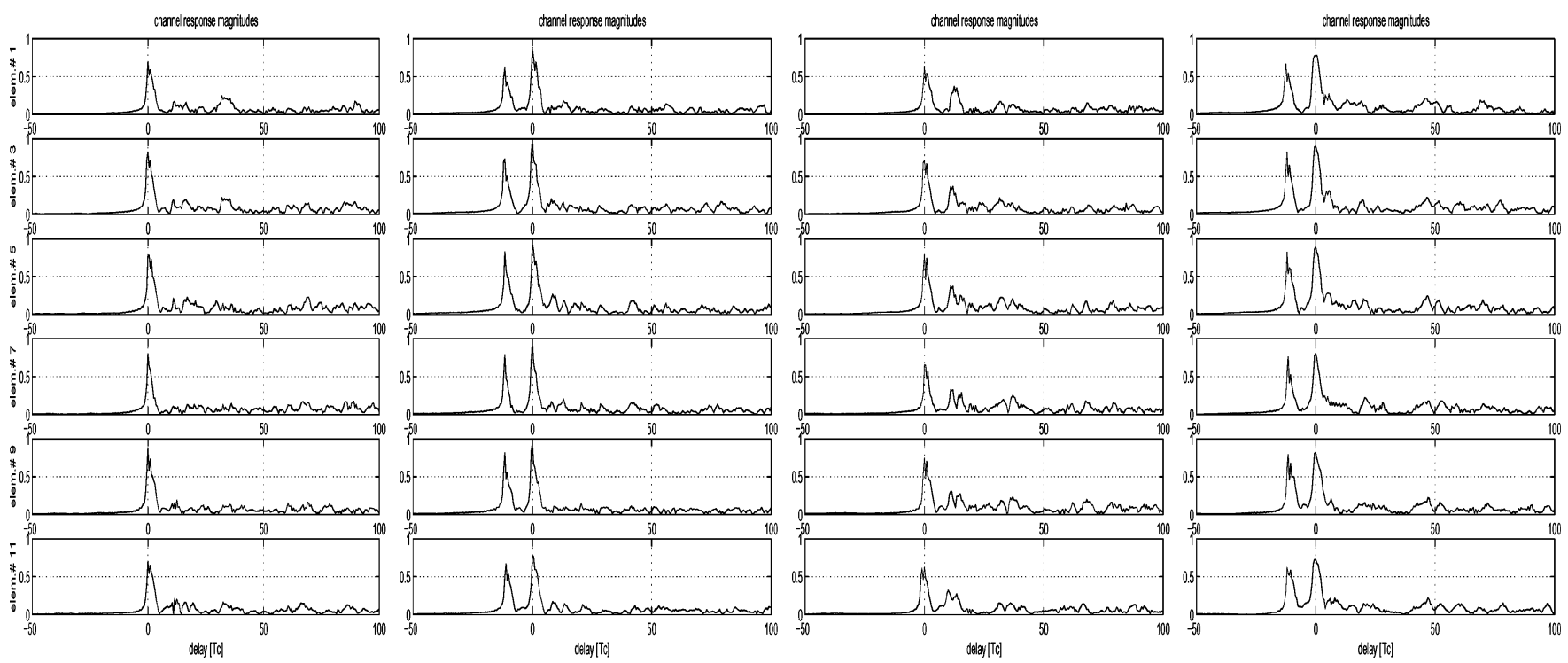

Fig. 6. Channel responses in space and time. Estimates are obtained by matched filtering to the frequency sweep channel probe, which is transmitted before each data packet. Each column shows the magnitudes of the channel responses estimated across the array, for every other of the 12 array elements spaced at $0.15 \mathrm{~m}$. The elements are numbered top to bottom, and each response is shown as a function of delay measured in chip intervals $\left(T_{c}=1 / R_{c} \approx 0.05 \mathrm{~ms}\right)$. The four columns correspond to the four users, whose packets are transmitted in sequence, separated by $24 \mathrm{~s}$. (The signals are later added to form the multiuser test signal.) This figure shows the channel responses recorded during transmission of signals with $L=255$ spreading factor.
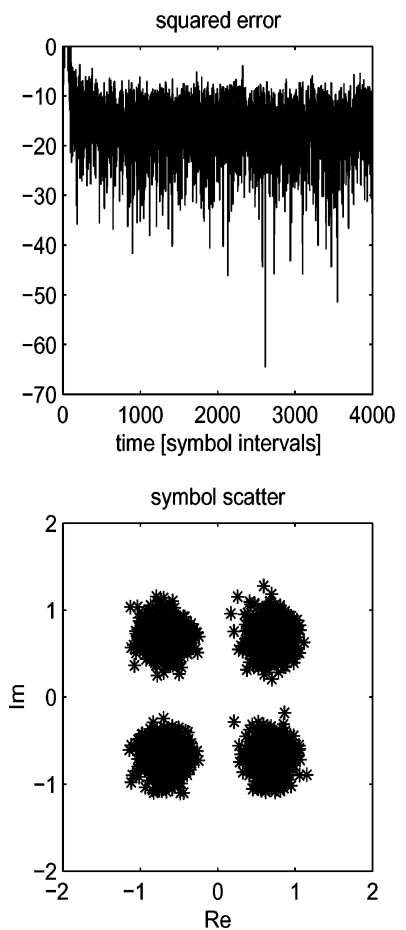

Fig. 7. Performance of the symbol decision feedback receiver with four users at the spreading factor of $L=15$. User 1 is the desired user; interfering users arrive asynchronously with relative delays of 8,4 , and 2 chip intervals. All the users have equal power.

and the output scatter plot of the data symbol estimates after training. Receiver parameters are listed in the figure, together with the number of training symbols $N_{t}$. Listed in the figure are also the Doppler frequency $f_{d}$ measured from the phase estimate, the ratio of erroneously detected symbols $P_{e}$, and the SNR measured from the output scatter plot. At $L=15$, the $\mathrm{Nc}=15$ chips/symbol $\mathrm{K}=12 \mathrm{P}=4$ channels $\mathrm{N}=6 \mathrm{M}=2$ (equalizer taps) LMS step size (equal.) $=0.005$ RLS forgetting factor (comb.) 0.992 $\mathrm{Kf} 1=0.0075 \mathrm{Kf} 2=0.00075$ $\mathrm{Nt}=160$ symbols $\mathrm{fd}=-1.3 \mathrm{~Hz}$

$\mathrm{Pe} 0$

SNRout $13.6 \mathrm{~dB}$
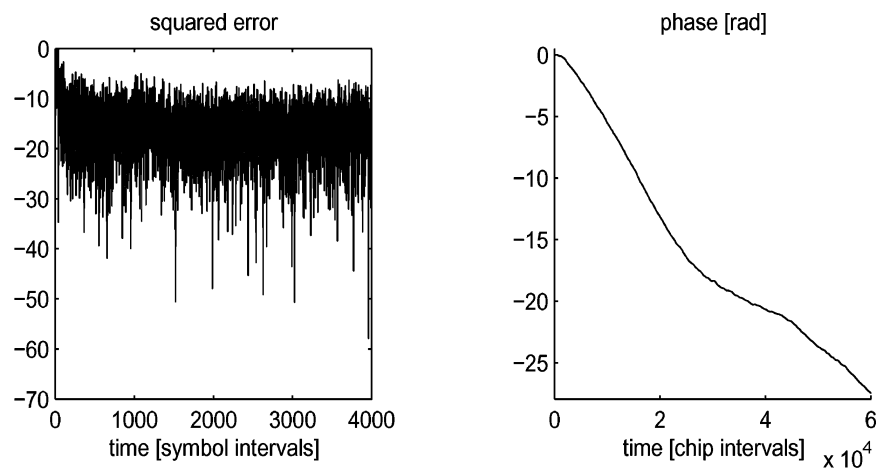

symbol decision feedback receiver:

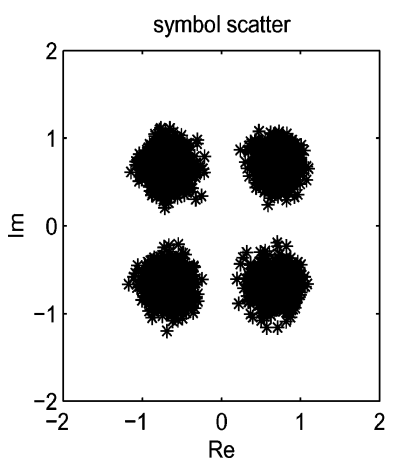

chip hypothesis feedback receiver: $\mathrm{Nc}=15$ chips/symbol $\mathrm{K}=12 \mathrm{P}=4$ channels $\mathrm{N}=6 \mathrm{M}=30$ (equalizer taps) LMS step size (equal.) $=0.0005$ LMS step size $($ comb. $)=0.05$ $\mathrm{Kf1}=0.0005 \mathrm{Kf} 2=5 \mathrm{e}-005$ $\mathrm{Nt}=160$ symbols $\mathrm{fd}=-1.4 \mathrm{~Hz}$ $\mathrm{Pe} 0$

SNRout $13.9 \mathrm{~dB}$

Fig. 8. Performance of the chip hypothesis feedback receiver with four users at the spreading factor of $L=15$. User 1 is the desired user; interfering users arrive asynchronously with relative delays of 8,4 , and 2 chip intervals. All the users have equal power.

performance of the SDF and the CHF receivers is similar. Very good results obtained at this lowest value of spreading factor owe to the use of multichannel combining. With a single array element, detection fails completely at $L=15$. At least six array elements were needed to ensure convergence. When all $K=12$ elements are used, $P=4$ short equalizers provide very good 

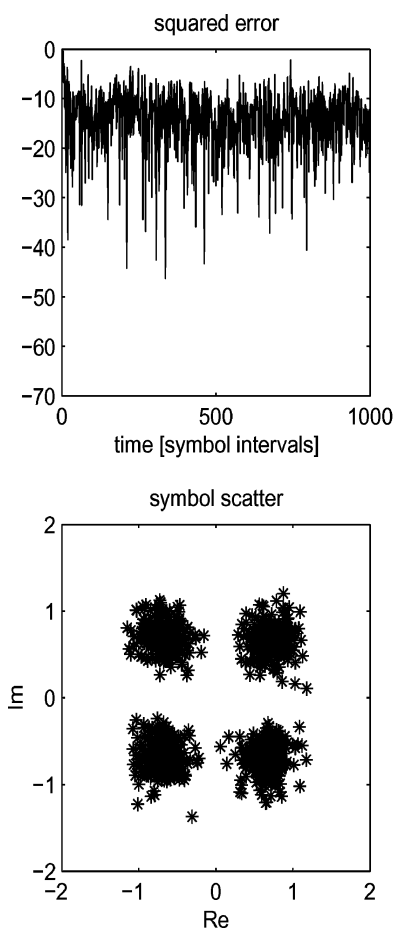

Fig. 9. Performance of the symbol decision feedback receiver with four users at the spreading factor of $L=63$. User 1 is the desired user; interfering users arrive asynchronously with relative delays of 8,4 , and 2 chip intervals. All the users have equal power.

performance. Each feedforward filter captures the principal arrival only using $N=6$ taps, while the feedback length of $M=$ 2 suffices to span the channel response when the SDF receiver is used. An alternative design with $P=2$ and $N=28$ provides similar performance. This result serves as a major encouragement as it demonstrates the possibility to support four users with minimal bandwidth expansion using a receiver of extremely low computational complexity.

Figs. 9 and 10 show performance at the spreading factor of 63. We recall that the channel at the time of this transmission appears more distorted than before. A longer feedforward section is chosen to capture both of the significant multipath components. The number of equalizers is now chosen to be $P=2$, which suffices for coherent array combining of the two principal arrivals. Both receivers provide very good performance; however, the advantage of hypothesis feedback becomes apparent as the spreading factor increases from 15 to 63 . Performance of the CHF receiver is about $4-5 \mathrm{~dB}$ better than that of the SDF receiver. Nonetheless, the SDF receiver provides very good performance, given its lower complexity of implementation.

The results obtained at the spreading factor of 255 are shown in Figs. 11 and 12. Although this is a high spreading factor to be used for multiuser support only, it is interesting to note the high quality of performance that can be obtained, and, thus, the margin left for the system if it needs to operate in higher interference or in the LPD regime. Almost identical performance is obtained for user 2, whose channel appears much more distorted. The data block now contains only 250 symbols; however, 10 symbols suffice for training. It may be somewhat surprising that the same low number suffices for the SDF receiver, the fact
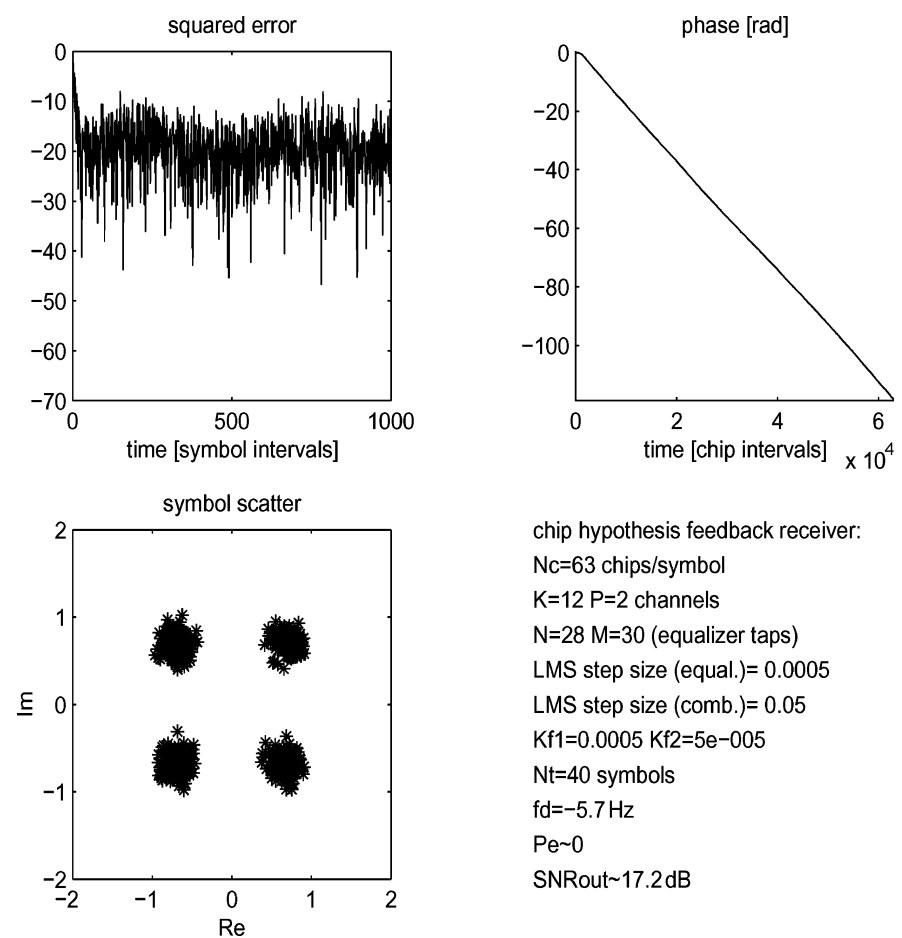

chip hypothesis feedback receiver: $\mathrm{Nc}=63$ chips $/$ symbol $\mathrm{K}=12 \mathrm{P}=2$ channels $\mathrm{N}=28 \mathrm{M}=30$ (equalizer taps) LMS step size (equal. ) $=0.0005$ LMS step size $($ comb. $)=0.05$ $\mathrm{Kf} 1=0.0005 \mathrm{Kf} 2=5 \mathrm{e}-005$ $\mathrm{Nt}=40$ symbols $\mathrm{fd}=-5.7 \mathrm{~Hz}$

Pe 0

SNRout $17.2 \mathrm{~dB}$

Fig. 10. Performance of the chip hypothesis feedback receiver with four users at the spreading factor of $L=63$. User 1 is the desired user; interfering users arrive asynchronously with relative delays of 8,4 , and 2 chip intervals. All the users have equal power.
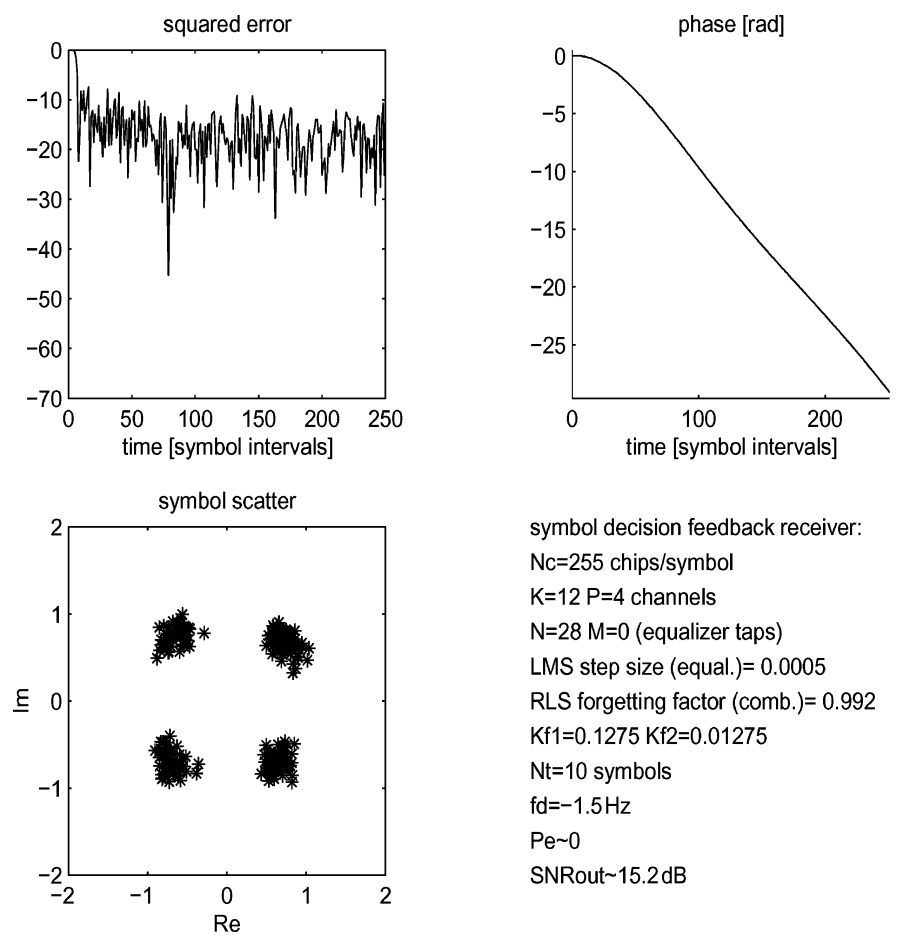

symbol decision feedback receiver: $\mathrm{Nc}=255 \mathrm{chips} / \mathrm{symbol}$ $\mathrm{K}=12 \mathrm{P}=4$ channels $\mathrm{N}=28 \mathrm{M}=0$ (equalizer taps) LMS step size (equal.) $=0.0005$ RLS forgetting factor (comb.) $=0.992$ $\mathrm{Kf} 1=0.1275 \mathrm{Kf} 2=0.01275$ $\mathrm{Nt}=10$ symbols $\mathrm{fd}=-1.5 \mathrm{~Hz}$ $\mathrm{Pe} \sim 0$ SNRout 15.2dB

Fig. 11. Performance of the symbol decision feedback receiver with four users at the spreading factor of $L=255$. User 1 is the desired user; interfering users arrive asynchronously with relative delays of 8,4 , and 2 chip intervals.

that is explained by high reliability that symbol decisions have at this spreading factor even before the receiver parameters have completely converged. Comparison between the SDF receiver and the CHF receiver reveals an advantage of $12-13 \mathrm{~dB}$, which 

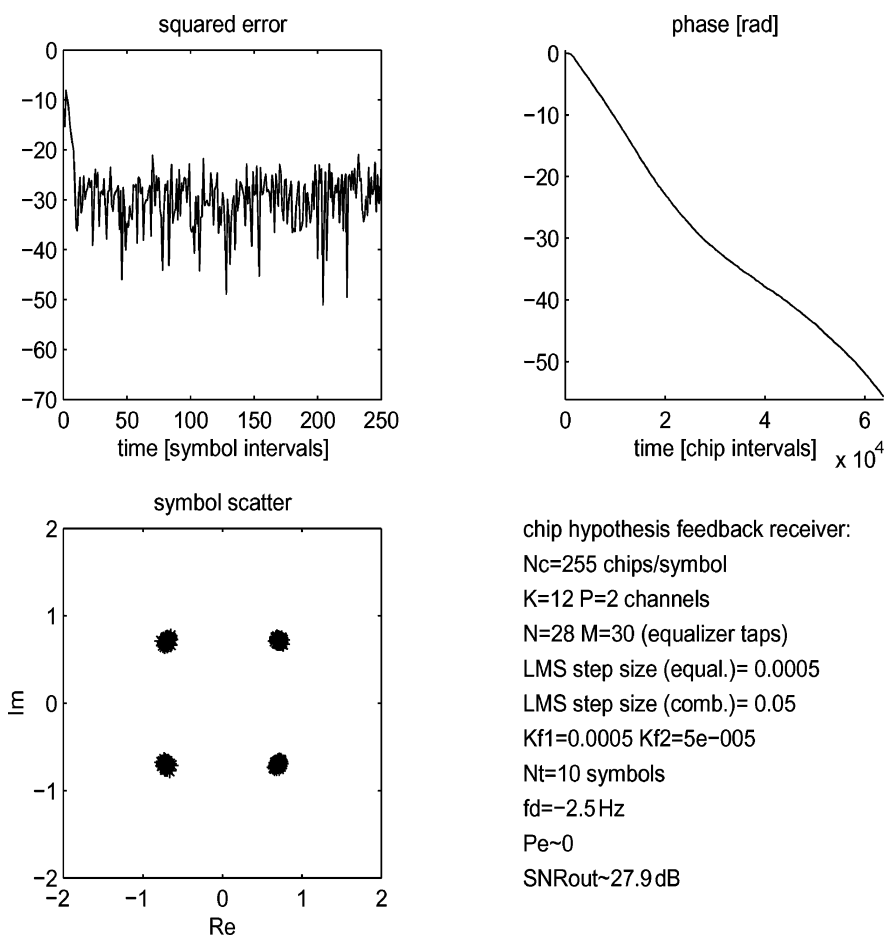

Fig. 12. Performance of the chip hypothesis feedback receiver with four users at the spreading factor of $L=255$. User 1 is the desired user; interfering users arrive asynchronously with relative delays of 8,4 , and 2 chip intervals.

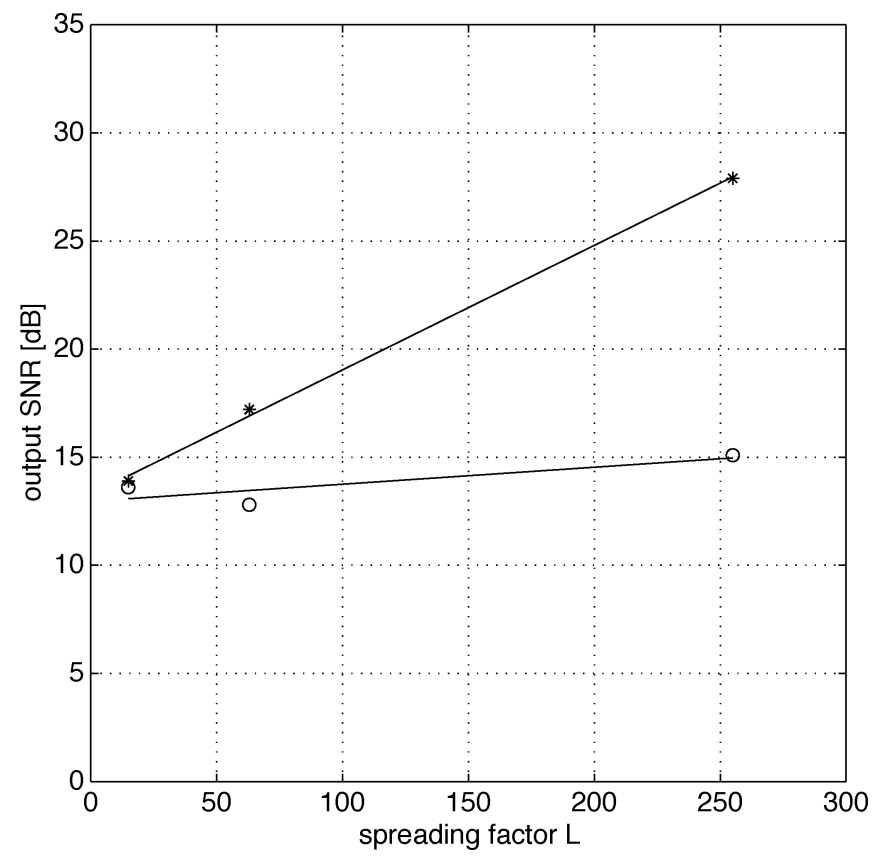

Fig. 13. Summary of experimental performance results: Output SNR for the $\mathrm{CHF}$ and the SDF receivers as a function of the spreading factor. Four equalpower asynchronous users at present in the system. The adaptive algorithm used for the combiner/equalizer is RLS/LMS for the SDF receiver and LMS/LMS for the $\mathrm{CHF}$ receiver.

results from the fact that the adaptation rate of the SDF receiver is now much slower than that of the $\mathrm{CHF}$ receiver, and also from the fact that the SDF receiver cannot take advantage of the feedback filtering.
In summary, Fig. 13 shows performance comparison of the two types of receivers as a function of the spreading factor. While the CHF receiver demonstrates consistent performance improvement with increasing $L$, the SDF receiver does not. This observation is in agreement with the fact that symbol-rate updating presents a tradeoff between tracking accuracy and interference suppression capability. Simulation results that state to the same effect for a Rayleigh fading channel and a Dopplershift-only channel are given in [10]. It must also be noted that the experimental data were obtained in stationary conditions. There was no intentional mobility in the system, only drifting (the estimated Doppler frequency of a few hertz implies a drift speed on the order of $0.1 \mathrm{~m} / \mathrm{s}$, which is in agreement with that observed during the experiment). If there were intentional mobility in the system, one could expect the SDF receiver to degrade more rapidly with an increase in the spreading factor. We thus conclude that for the shallow-water conditions of interest, the SDF receiver represents an excellent choice for a CDMA system operating with few users at a low spreading factor. When symbol-rate adaptation and feedback filtering are sufficient, this receiver offers minimal complexity of implementation. However, if a greater spreading gain is desired (and the bandwidth expansion can be afforded) the CHF receiver offers better performance, albeit at higher complexity. In an underwater acoustic system operating in shallow water over distances of a few kilometers, it is not likely that the number of users will justify a spreading factor as large as 255 . However, a large spreading factor may be desired to support LPD communication between several users. In this situation, $\mathrm{CHF}$ receiver should be chosen over the SDF receiver.

Finally, it should be mentioned that further reduction in computational requirements of the $\mathrm{CHF}$ receiver is possible through early discarding of some hypotheses. Namely, with a large spreading factor there may be no need to wait for the entire code period before making a decision. Partial despreading can be performed instead, and those hypotheses that appear to be wrong, i.e., whose partial MSE fails to increase above a certain level, can be discarded, thus reducing the amount of computations per symbol.

\section{CONCLUSIONS AND FURTHER WORK}

DS spread spectrum CDMA is a candidate multiple-access technique for a class of future mobile underwater acoustic networks. A particular scenario of interest is that of several AUVs operating in a wideband regime over a distance of a few kilometers to the central receiver, which can either be a fixed or a movable platform equipped with a receiving array for detection of asynchronous multiuser signals. For such a network, two types of multichannel detection algorithms for DS CDMA signals were designed: SDF and CHF receivers. The algorithms were tested using experimental data. Excellent performance was demonstrated, which owes to the integration of adaptive multichannel combining into the detection algorithms.

The SDF receiver represents a minimal complexity solution and it is suitable for systems where the channel variation can be tracked at the symbol rate. In a wideband acoustic system, where full bandwidth utilization is accomplished by transmitting at maximal chip rate, the symbol rate is down-scaled by 
the spreading factor. The spreading gain, and, consequently, the interference rejection capability, may thus be limited by the receiver's ability to track the channel at a symbol level. Channel variation is mostly influenced by the system mobility, and it can become a critical issue in a wideband AUV system. In such a situation, the CHF receiver should be used, as it performs chip-rate adaptation. At the price of increased computational complexity (but still only linear in the modulation level) this receiver provides improved performance, which consistently increases with the spreading factor used. A large spreading factor may not be necessary in a small CDMA system; however, it is of interest to LPD systems. In the experimental study, using a chip rate of 19.2 $\mathrm{kc} / \mathrm{s}$, it was found that the two receivers provided comparable performance at the spreading factor of 15 , while as the spreading factor increased to 63 and 255, the CHF receiver gained considerable advantage.

Both of the receivers proposed offer a realistic platform for a next generation system that needs to support wideband acoustic CDMA communications. While the experimental results of this paper may influence the selection towards the SDF receiver because of its extremely low complexity, it must be kept in mind that these results were obtained using a stationary system. Future field tests should encompass a broader range of conditions, including transmission from moving AUVs, with spatially diverse multiuser channels.

\section{REFERENCES}

[1] E. M. Sozer, M. Stojanovic, and J. G. Proakis, "Underwater acoustic networks," IEEE J. Ocean. Eng., vol. 25, no. 1, pp. 72-83, Jan. 2000.

[2] D. Kilfoyle and A. Baggeroer, "The state of the art in underwater acoustic telemetry," IEEE J. Ocean. Eng., vol. 25, no. 1, pp. 4-27, Jan. 2000.

[3] M. Stojanovic, J. Catipovic, and J. Proakis, "Reduced-complexity multichannel processing of underwater acoustic communication signals," J. Acoust. Soc. Amer., vol. 98, no. 2, pt. 1, pp. 961-972, Aug. 1995.

[4] M. Stojanovic and Z. Zvonar, "Multichannel processing of wideband multiuser communication signals in shallow water acoustic channels," IEEE J. Ocean. Eng., vol. 21, no. 2, pp. 156-166, Apr. 1996.

[5] G. Woodward and B. Vucetic, "Adaptive detection for DS-CDMA," Proc. IEEE, vol. 86, no. 7, pp. 1413-1434, Jul. 1998.
[6] M. Abdulrahman, A. Sheikh, and D. Falconer, "Decision feedback equalization for CDMA indoor wireless communications," IEEE $J$. Sel. Areas Commun., vol. 22, no. 4, pp. 698-706, May 1994.

[7] J.E. Smee and S. C. Schwartz, "Adaptive space-time feedforward/feedback detection for high data rate CDMA in frequency-selective fading," IEEE Trans. Commun., vol. 49, no. 2, pp. 317-328, Feb. 2001.

[8] J. F. Rossler, L. H.-J. Lampe, W. H. Gerstacker, and W. H. Huber, "Decision-feedback equalization for CDMA downlink," in Proc. Veh. Technol. Conf. (VTC), May 2002, vol. 2, pp. 816-820.

[9] A. Santoso, J. Choi, and B. Vucetic, "Chip level decision feedback equalizer for CDMA downlink with space-time coding," Electron. Lett., vol. 7, no. 7, pp. 437-439, Apr. 2004.

[10] M. Stojanovic and L. Freitag, "Hypothesis-feedback equalization for direct-sequence spread spectrum underwater communications," in Proc. IEEE Oceans'00 Conf., Sep. 2000, vol. 1, pp. 123-129.

[11] F. Hasegawa, J. Luo, K. Pattipati, P. Willett, and D. Pham, "Speed and accuracy comparison of techniques for multiuser detection in synchronous CDMA," IEEE Trans. Commun., vol. 52, no. 4, pp. 540-545, Apr. 2004.

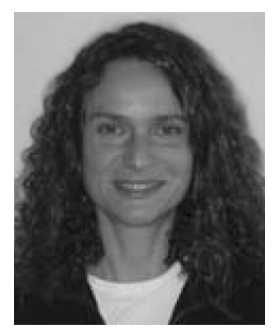

Milica Stojanovic (S'90-M'93) graduated from the University of Belgrade, Yugoslavia, in 1988 and received the M.S. and Ph.D. degrees in electrical engineering from Northeastern University, Boston, MA, in 1991 and 1993, respectively

She is currently a Principal Scientist at the Massachusetts Institute of Technology, Cambridge, and also a Guest Investigator at the Woods Hole Oceanographic Institution, Woods Hole, MA. Her research interests include digital communications theory and statistical signal processing, and their applications to mobile radio and underwater acoustic communication systems.

Dr. Stojanovic is an Associate Editor for Communications with the IEEE Vehicular Technology Society.

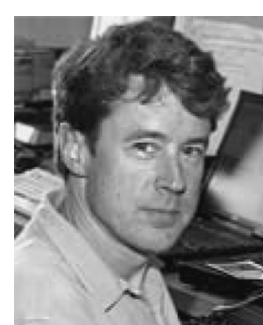

Lee Freitag (M'88) received the B.S. and M.S. degrees in electrical engineering from the University of Alaska, Fairbanks, in 1986 and 1987, respectively.

$\mathrm{He}$ is currently a Senior Engineer at the Woods Hole Oceanographic Institution, Woods, Hole, MA, where he has worked on projects related to underwater acoustics for 15 years. His research programs focus on underwater acoustic communication and navigation with a strong focus on unmanned underwater vehicles (UUVs), sensors, and submarine systems. 